Research Article

\title{
Motion Attitude Recognition and Behavior Prediction Algorithm Based on Wireless Sensor Network
}

\author{
Tianping Zhang, ${ }^{1,2}$ Bo Zhang $\mathbb{D}^{2},{ }^{2}$ Lei Liu, ${ }^{2}$ and Yang Liu ${ }^{2}$ \\ ${ }^{1}$ School of Logistics Engineering, Wuhan University of Technology, Wuhan 430070, Hubei, China \\ ${ }^{2}$ School of Computer Science, Wuhan Donghu University, Wuhan 430212, Hubei, China \\ Correspondence should be addressed to Bo Zhang; zhangbo20210405@163.com
}

Received 27 August 2021; Revised 4 October 2021; Accepted 15 October 2021; Published 27 October 2021

Academic Editor: Sang-Bing Tsai

Copyright (C) 2021 Tianping Zhang et al. This is an open access article distributed under the Creative Commons Attribution License, which permits unrestricted use, distribution, and reproduction in any medium, provided the original work is properly cited.

\begin{abstract}
The wireless sensor network is an integral part of the physical information system. Disperse sensors through a set of special spaces track and record the natural state of the environment and manage the information collected in a central location. The sensors use wireless connections to create their own networks. Wireless sensor network technology has the advantages of flexible deployment and convenient use and has played an important role in the field of user behavior recognition. By deploying wireless sensor network technology, users can collect daily information, capture users' behavior habits, and analyze users' health status. In the deployment and application of this type of technology, it is very important to build an effective model of the logical sequence relationship of the monitored person's behavior. The sensor data can be sent to the target user through wireless transmission. Action recognition is often based on a single feature for learning and judgment, so there are many difficulties in practical applications. This article aims to study motion shake awareness and action prediction algorithms based on wireless sensor networks. Aiming at the research of human pose recognition algorithm, to optimize the overall performance of the model, this article suggests the use of multimodal input, uses a 2D and 3D network structure, and finally, proposes two network weighted fusion strategies. Aiming at the research of pedestrian motion discrimination, this article offers a behavior prediction algorithm based on multifeature joint learning. The algorithm adds the feature vectors output by gesture recognition and mask prediction and uses a cross-entropy cost function to jointly learn and predict classification. The results of the survey show that the pedestrian gesture recognition and motion recognition algorithm based on the wireless sensor network proposed in this paper has good performance and can be widely used in real scenes such as video surveillance. The accuracy of the gesture recognition algorithm in the UCF101 dataset and the HMDB51 dataset was $96 \%$ and $72 \%$, respectively.
\end{abstract}

\section{Introduction}

Recently, with the development of wireless sensor networks and microelectronics device technologies, first, the underlying raw data is preprocessed, and then, the activity recognition analysis is performed. Finally, on the basis of these data, we conduct content analysis such as mode and intent. At present, there are many applications for behavior recognition. For example, the location service architecture provided by Android can automatically recognize the user's real-time physical activities, such as walking, standing, and running, and also calculate the probability of each activity. With the development of science and technology, the degree of clustering and miniaturization of sensor nodes is getting higher and higher, so that sensor nodes can develop in special or dangerous environments inaccessible to humans. But in terms of the power of sensor nodes, there is not yet a full range of renewable energy technologies. When the size of a sensor node decreases, the power delivered to the node is limited. However, developed sensor nodes are often difficult to provide backup power. Therefore, how to extend the network lifetime is a necessary factor in the design of wireless sensor routing protocols, but the power balance of the entire network must also be considered to avoid the problem of "hot spots" in the network. Most wireless sensor networks are composed of sensor nodes and assembly nodes, 
which collect, calculate, and send target data to the monitoring area. A wireless sensor network that can communicate with the outside world also has a base station, midrange network equipment, task management system, and gateway. The wireless sensor network (WSN) is a distributed network system that collects information by a series of miniature sensor nodes and transmits information by means of wireless communication. Sensor networks are widely used in industry, medical and health, agriculture, transportation, and other fields, such as monitoring various physiological data of the human body, hospital drug management, and so on.

In recent years, the continuous progress of machine learning and computing technology has promoted the rapid development and wide application of smart home technologies. Smart home technology can realize functions such as remote control of home appliances and monitoring of living environment conditions by arranging wireless networking sensor nodes in the environment where users live. In recent years, digital image processing technology and computer vision technology have been continuously developed and advanced. On this basis, the detection and tracking technology of moving targets has also made considerable progress, and experts and scholars have continuously proposed new theories and algorithms. However, corresponding to the progress in theory, the technology is very complicated in its realization. At present, many technical aspects still need to be improved, and there are still many problems that need to be solved in practice. For example, in a very complex environment, the real-time performance and reliability of the algorithm are compromised in the implementation process. Therefore, research on target detection and tracking technology not only has strong theoretical value but also has great potential commercial value and application prospects. At present, it has attracted a lot of scientific researchers and research institutions. The research has great practical significance. Video surveillance needs to be watched by a dedicated person in real time, which may infringe on the user's personal privacy. In addition, the staff responsible for watching and monitoring are prone to fatigue after long-term work and may not be able to deal with emergencies in time. For another example, video surveillance personnel will inadvertently spread the content of the user's video, which seriously violates the user's privacy. User behavior recognition based on wireless sensor networks can not only reduce the risk of privacy information leakage but also effectively save the human resources required for monitoring.

In the professional medical field, user behavior recognition technology based on wireless sensor networks can also be widely used. In view of the shortage of medical staff, behavior monitoring can be carried out in scenes such as emergency rooms or operating rooms, so as to realize the reasonable transfer of personnel. Or when conducting scientific experiments, the experiment process can be monitored in real time, so that dangerous situations can be avoided. In the factory environment, construction personnel can be monitored in real time, so that the entire work process can be monitored in real time, which facilitates the detection and identification of products. Human motion detection and gesture recognition are to directly use the computer to analyze and process the visual information of the video, detect the moving human body and the posture in the video, and describe and express the information and purpose of the posture. With the development of computer technology and information technology, society needs more demanding video analysis. It is hoped that the human body posture information can be directly obtained through computer analysis of the video. Because of its deeper understanding of the human visual system, human body posture research is one of the key technologies to be finally realized in the field of wireless sensors. Moving human body gesture recognition takes digital image processing, pattern recognition, and other technologies as the key. On the basis of image information, the detection, extraction, and recognition operations are realized through analysis, and whether it is abnormal is judged. Human body gesture recognition has a wide range of applications and has great social and economic value.

Zhu's team believes that machine learning technology is becoming increasingly important for the upcoming paradigm shift to data-intensive science. In particular, deep learning has proven to be an innovative and extremely powerful tool in many areas [1]. The research of Bansal's team found that, with the increasing use of computers in our daily lives, the work of developing better human-computer interaction interfaces has rapidly increased. Now more than ever, there is a need for an easy-to-use advanced humancomputer interface. In this framework, the computer's UI allows users to interact with electronic devices through graphical icons and visual cues, which is still inconvenient and unsuitable for working in a virtual environment. Interfaces that allow users to communicate through gestures are the next step in the direction of advanced human-machine interfaces [2]. Shen's team proposed a new MC-CNN model to classify the suspiciousness of lung nodules. Their MC-CNN extracts the salient features of multiscale nodules in a little network. The method they proposed does not respond to nodule segmentation and once-made characters [3]. The wireless sensor network is considered to be one of the most promising technologies because of its advantages such as continuous monitoring, flexible deployment, and low cost. In addition, the inherent nonintrusive characteristics of these networks have been proven to be suitable for environments that require privacy protection. Previous methods have proved that simple binary sensors have good potential in solving the problem of family ADL recognition and can be applied to health care problems centered on the elderly.

\section{Proposed Method}

2.1. Posture Recognition Algorithm. Movement pose recognition is the extraction, classification, and natural language description of human posture characteristics. It is a widely concerned research topic in recent years. It is a research field of various disciplines such as applied human physiology, digital image processing, pattern recognition, 
and other disciplines. To overcome the limitations and uncertainty of taking the classification results of single frames as the final identification result, we propose a quadratic classification algorithm based on image similarity. Based on the first single-frame-based SVM classification, the algorithm adaptively assigns the statistical weights of the first classification results within the discriminative period by calculating two adjacent image frames, performs quadratic pose classification, and finally outputs the pose statistical results within a discriminative period. The algorithm increases the degree of correlation between front and posterior video frames in the recognition process and improves the accuracy of posture recognition while ensuring real time.

Posttitude feature extraction of human object images is a two-dimensional array. Feature extraction of human targets into one-dimensional feature vectors can greatly reduce the eigenvector dimension and computational complexity. This paper is mainly for walking, running, and jumping, by analyzing the three postures in arm swing amplitude and leg stride amplitude, using star model for three-human modeling, that is, by calculating the movement body target image centroid of mass and local contour pole and its relationship, in order to obtain posture characteristics. Perfect description of human contour includes the head and limbs and is not redundant.

How to determine the motor human characteristics to be extracted, generally corresponding to the movement type of the motor target, the main requirements for the characteristics have the following three points:

(1) Can fully show the movement characteristics of the human body

(2) Should have different characteristics and should not be too similar

(3) Can have more than one feature of the same monitoring object, but it cannot be associated between each feature

2.1.1. Multistream Convolutional Neural Network. Using RGB data to extract scene, appearance, and other information, RGB data will be used as input for both $2 \mathrm{D}$ convolutional and $3 \mathrm{D}$ convolutional jobs $[4,5]$.

The multistream convolutional neural network is divided into 5 network branches, three of which are two-dimensional convolutional neural networks. InCeption-V3 is used as the basic network, and the attention structure is embedded in the network structure to allow the network to learn key areas autonomously. Features reduce noise interference $[6,7]$. At the same time, the network contains three modal data inputs, namely, single-frame RGB data, single-frame gradient map data, and stacked dense optical flow map, to maximize the time and space information in human behavior recognition $[8,9]$. The other two branches are three-dimensional jobs. The data input of the threedimensional job is continuous multiframe RGB map and gradient map data $[10,11]$.
2.1.2. Network Fusion Based on Weight. The wireless sensor network is a cutting-edge hot research field widely concerned, interdisciplinary, and highly gathered knowledge at home and abroad. The wireless sensor network consists of multiple sensor nodes across different regions, forming a network of self-service through wireless communication that collaborate with each other and monitor, perceive, and collect various pieces of information about the monitoring objects and environment in real time. With the development of sensor node technology, the wireless sensor network has been applied to environmental monitoring, national defense and military affairs, medical and health care, transportation planning, and other fields, and its influence cannot be ignored. However, the throughput, bandwidth, and energy of wireless sensor networks, etc., are very limited, and how to effectively utilize storage and computational resources has become the bottleneck of sensor network design. Data fusion technology is an important technology to achieve energy saving by removing redundant methods and thus improving energy use efficiency. In the wireless sensor network, sensor nodes have a wide variety of characteristics, dense layout, collaborative perception, and data acquisition process. Adjacent nodes through the collected data have similar places. In practice, we only focus on its results and do not need to collect a lot of original information. So in information collection, we make full use of node-local computing ability and storage ability, a number of pieces of information or data processing, and combined effective information. This processing is data fusion.

(1) Network Fusion Based on Model Weights. First, we assume that the weight space is three-dimensional space $R^{x y z}$; that is, there are three kinds of network branches. We set the length boundary of each dimension to $x, y$, and $z$; you need to find a set of optimal weight combinations $(u, v, w)$ in this two-dimensional weight space as the weight of the network model.

We traverse the weight space, where the sliding steps of each dimension are $p, q$, and $k$, respectively, to obtain all possible weight values for each dimension. The weight with the highest accuracy is used as the final network model weight $[12,13]$.

The final classification score is shown in formula (1), where $w$ represents the weight value of each dimension in the weight space and $s$ represents the classification score obtained by each network. In the fusion of classification scores, after weighing each score, there are two decisionmaking methods: average and maximum, namely, $F$ function in formula (1). In this paper, after the experimental comparison, the decision method of averaging is adopted.

$$
\text { score }=\max \left(F\left(w_{x} S_{1}, w_{y} S_{2}, w_{z} S_{3}\right)\right) .
$$

(2) Network Fusion Based on Category Weight. Due to differences in data between behavior types, different networks have different classification effects on these behaviors $[14,15]$. First, we calculate the sum of the accuracy rates of all network models in each category, then find the proportion of 
the accuracy rate of each model to the total accuracy rate, and finally multiply the number of network models to normalize the weights to $[0,1]$. As shown in formula (2), for the network model $k$, the weight of the behavior category $j$ is

$$
\bar{w}_{k}^{j}=\frac{a_{k}^{j}}{\sum_{i=1}^{n} a_{i}^{j}} * n .
$$

Among other things, representing the network model number, we represent the isolation standard in the validation set. Finally, after obtaining the weight of each category of the network model $k$, the classification score of the network model's behavior category inference is calculated as shown in formula (3), where $S$ represents the behavior category inference score of the original network model, and $W$ represents the network model the category weight; $\otimes$ means elementwise multiplication.

$$
\text { score }=S \otimes W_{k} .
$$

(3) Background Split Method. Background differential method is a common detection method of motion object, also known as background cutting method. The advantage of the background difference method is that it can extract a relatively complete target area, but the corresponding background difference method is too sensitive to external changes. If the light or shadow changes, it will cause errors in the initial background image, resulting in the final detection The accuracy of the moving target is reduced, and the extraction effect is also very poor.

(4) Frame Difference Method. In the static background, because the target is in the motion process, there will be some differences between the two frames of images in the continuous image sequence. Through the detection of this difference, the motion object can be detected and extracted, which is the difference algorithm between adjacent frames. The advantage of the interframe difference method is that the algorithm is simple and easy to implement, which is very suitable for application in real-time motion target detection, and does not need to consider the background update. Because the time difference between frames and frames in the continuous frame video sequence is small, the light change has less impact on the results of the algorithm, and the adaptability of the shadow is stronger than the background difference method, which is more practical for the change of the external environment.

The goal of motion gesture recognition is to divide the movement-generated area from the background of the video image, clarify the target location and size, simplify the video processing process, and facilitate the extraction of the later movement information, the tracking, and identification of the target. In practical scenes, many factors will affect the accuracy and segmentation effect of motion posture recognition, such as external condition factors including the change of light, the shaking branches, the occlusion of objects, and the influence factors of the hardware equipment including the jitter and noise of the camera. Therefore, the corresponding algorithms should be adopted for realistic scenarios and system requirements $[16,17]$.

Nowadays, the main difficulties in human motion posture recognition based on wireless sensor networks are as follows:

(1) In the research field of wireless sensors, target detection and tracking are the basis of human posture recognition, but in practice, the problems in the early preprocessing work cannot be usually well solved. In the process of video image acquisition, many practical factors, such as lighting, occlusion, shading, point of view, and camera equipment, will affect the early processing effect, thus reducing the accuracy of posture recognition.

(2) Good motion gesture profile description method is a prerequisite for motion gesture recognition. The uncertainty of movement posture leads to the difficulty to achieve uniform division indicators, which increases the difficulty of motor posture characteristics.

(3) The ultimate purpose of movement posture analysis is to understand the information conveyed by people's movement posture, which requires not only identifying the basic movement posture but also requires comprehensive analysis combined with scene information. A large number of studies are limited to regular motion poses of simple categories, and the analysis of specific motion poses in specific scenarios remains to be further investigated.

2.2. Behavior Prediction Algorithm. This paper proposes a prediction framework for feature fusion based on behavioral prediction algorithms. In behavioral prediction algorithms, a single feature expression does not cope well with problems such as background interference in the video. Based on this, this paper designs a feature fusion framework based on sparse self-coding to effectively integrate motion features (multiscale optical histogram) and apparent features (significant information), not only providing dimensionality reduction to remove redundancy but also generating richer combined features and high-level features.

We enter a picture to be detected; after the first stage, you can get the coordinates of the joints and the character mask of each person in the picture, and then we do coordinate normalization and cutting to get the normalized coordinates and the cut image $[18,19]$. In the second stage, the normalized coordinates are sent to MLP and the cut image is sent to ResNet to extract local features and global features $[20,21]$. Then, the obtained features are combined and allocated to a single network for isolation. Here, the crossentropy compensation function is used to find the model gradient:

$$
C=-\frac{1}{m} \sum_{i=1}^{m} y^{i} \cdot \log \left(h^{i}\right),
$$


where $M$ is the number of batches of the current training data, $y^{i}$ is the number of distribution, that is, the one-hot encoding of the label, and $h^{i}$ is the predicted probability distribution.

The algorithm training process in this paper is trained in two steps:

(1) The first stage of training: the original feature extraction network VGG-19 uses the first five layers of convolution kernel initialization of the open-source ImageNet classification model, and the remaining new layers use the parameter initialization method Xavier initialization to make the convolution kernel weights follow the following distribution $[22,23]$ :

$$
W \sim U\left(-\sqrt{\frac{6}{n_{\text {in }}+n_{\text {out }}}}, \sqrt{\frac{6}{n_{\text {in }}+n_{\text {out }}}}\right) .
$$

(2) The second stage of training: the use of Xavier initialization to initialize the weight $w$ of the MLP is to help the signal pass deeper in the neural network, so that the signal remains within a reasonable range after passing through multiple layers of neurons; the offset $b$ of the MLP often initializes the offset to 0 , and floating-point initialization will cause weight imbalance.

ResNet also uses the Xavier initialization parameter initialization method, but because the training speed of large convolutional networks needs to be improved, the famous batch normalization method (Batch Normalization, BN) is used. We normalize the coordinates of the joint points in the rectangular area, and the coordinates are

$$
\left(\frac{x_{i}-x_{o}}{w}, \frac{y_{i}-y_{o}}{h}\right) \text {. }
$$

A large amount of behavioral data is collected, real-time tracking training of the learning algorithm realizes the prediction of the next behavior of people, and the accuracy is very high. Although, compared with other learning cases, there is no complete replacement behavior, the next behavior is more accurate. The algorithm uses the migration learning method to effectively solve the characteristics of new field learning and assists the target organization training with the source tissue samples and iteratively corrects the false sample weights in training, so as to overcome the problem of lack of samples causing prediction difficulties.

\section{Experiments}

This paper mainly studies the attitude recognition of people in the family environment, including movement object detection from low to high measurement, target tracking, and attitude recognition algorithm. We first introduced several commonly used motion object detection algorithms, including the between frame difference method and simulation, through morphological processing and regional analysis to filter out the acquisition image noise. Finally, we extract the target external outline and center of mass coordinates. In the aspect of motion target tracking, the accuracy of posture recognition is improved through the introduction and comparison of the current common tracking methods. Finally, the movement object feature analysis method is adopted to realize the identification and detection of the moving body, mainly by judging the aspect ratio change and the displacement of the centroid coordinates.

3.1. Data Collection. There are many sensors in the equipment, including transmitters, gyroscopes, angular velocity meters, pressure sensors, etc. Data collection uses sensors to collect information about different actions performed by the human body. The action information in the process of completion is converted into electrical signals and uploaded to realize simple logic operations, data storage, and communication requirements. According to the requirements of actual applications, the sensor module can meet the work requirements, the information simulation required in human body recognition, and the internal analysis and processing of the node need to be completed, so the design of the node needs to include multiple sensor modules, which can be used in conjunction to complete the work of the system requirement. Under normal circumstances, a node contains four modules, consisting of a processor module, a power supply, a sensor module, and an intelligent module. The processor module is composed to control the normal operation of each functional module of the sensor node and perform the related processing of each signal; the sensor module realizes the function of detecting the movement information of the object and realizes the transmission of information to electrical signals; if the communication module overloads the signal, the data is transmitted wirelessly to other devices; the power supply provides the energy for its normal operation. The function of the upcoming sensor node is to collect signals at critical moments in the human body. When it occurs with the sensor node, the location of the mobile device is not fixed, and the recognition result of the system will have an impact. The sensor detects motion information, and the device can be placed in a fixed location. The location avoids this effect. There are many platforms that the human body finds at present. Researchers need to build a posture detection platform according to their own research needs.

All experiments in this section were performed on the UCF101 dataset and the HMDB51 dataset. The UCF101 dataset has a total of 13320 videos, including 101 categories, and each category has about 100 videos. Among them, the coach database includes 9537 videos and the experimental database includes 3783 videos. The HMDB51 data set has a total of 6,766 videos, including 51 categories, and each category has at least 101 videos. The training data of each category is 70 videos, and the test data is 30 videos.

3.2. Environment of Survey. The test development area is the Linux system, and the graphics card is NIVDIA's Teslak40m model. The software development environment used includes deep learning frameworks such as MXNet library, image processing library OpenCV 2.4.13, parallel computing platform Cuda9.0, and GPU acceleration library Cudnnv7.0. 
3.3. Training Parameter Settings. The gradient method is used for optimization, and the level is set to 32 . The twodimensional convolutional network model that introduces the attention mechanism uses cross-entropy loss function, the initial training rate of the RGB network-based gradient and gradient data is set to 0.005 , the learning rate is set using the step method, and the step rate is set to 2000. After 2000 iterations, the learning rate drops to one-tenth of the previous value. The initial learning rate of the optical flow-based time flow network is set to 0.001 , and the learning rate is adjusted using the multistep method. When it reaches 10,000 and 16,000 iterations, the learning rate drops to one-tenth of the current value, and the maximum number of iterations is set to 20000. The replaced 3D network input data is generally cut into continuous multiframe data of $112 * 112$. The model uses a cross-entropy loss function, the initial training rate is set to 0.001 , and the step method is used to set the curriculum rate, which is 3000 times for renewal learn to a quarter of the previous value, and the maximum number of configurations is set to 10000 .

\section{Discussion}

\subsection{Study of Network Experiment Results}

4.1.1. Study of Network Fusion Experiment Results. Most of the occurrence of human behavior is irregular. Different people will have different manifestations of the same behavior. When one behavior occurs, other behaviors may happen. For example, when the user's behavior is sleeping, he may get up to go to the toilet halfway through, wash his hands, etc. For example, the user can watch TV and make phone calls while cooking. Behavior recognition is uncertain, and the process of many behaviors is the same, which will make the collected sensor data almost the same. The staggered concurrency of behaviors makes behavior identification challenging.

For all network fusion methods, that is, average fusion, model-weighted fusion, and category-weighted fusion, three experiments have been carried out to compare and verify. Weighted fusion works the best. Furthermore, when both model weighting and category weighting are applied, the classification accuracy of the model is the highest. The results of the network integration experiments are shown in Table 1 and Figure 1.

Behavior recognition data is usually collected by indoor sensors, and the actual collected data is affected by noise, and the data is also inaccurate. The types of behaviors that the behavior recognition model can recognize are limited. The collected data contains sensor data segments corresponding to the observed behaviors, which contains many empty data segments, which easily interfere with the recognition results. The existence of empty data makes behavior recognition require special data preprocessing to remove useless data.

4.1.2. Differences in Classification Results between Different Networks. Human behavior will be affected by many factors, such as pressure, emotion, and environment. The same behavior of the same user will also have different performances. The traditional method of user behavior recognition is based on the similarity of the same type of behavior. Due to the diversity of peer behavior, it is necessary to make the recognition model more antiinterference.

Part of the experimental results on the UCF101 dataset is as follows: for the behavior of drawing eyeshadows and blowing hair, the network classification based on RGB data is better; for the behavior of playing golf and shooting, the network classification based on optical flow data is better; for flute playing and hair cutting, the network classification based on gradient data is better. The classification results between different networks are shown in Figure 2.

4.2. Analysis of Experimental Results of Motion Pose Recognition and Behavior Prediction Algorithms. There is a difficulty in the realization of movement posture recognition, due to the change of the target movement state or other interference factors, which will reduce the accuracy. In order to solve this problem, we can estimate the movement target information, in order to accurately achieve movement target tracking, through the analysis of different gesture recognition algorithms.

\subsubsection{Analysis of Comparison Experiment Results of Different} Motion Gesture Recognition Algorithms. However, it seems that everyone's test data and types of behavior are different, and it is difficult to compare. In addition, most of the modeling of sensor data and user behavior is carried out on the traditional probability model. The traditional probability model has a good theoretical foundation, but it also has a lot of limitations. For this point, this paper proposes a hybrid model, which relieves the assumptions of the traditional probability model, and proposes a method to determine the initial parameters of the behavior model.

So far, based on the human behavior recognition in the article, the results of the baseline algorithm are compared. Compared with the traditional IDT algorithm, dual-stream convolutional neural network, C3D, and other algorithms, the multistream convolutional neural network algorithm proposed in this paper has a great improvement. The comparison experiment results of different motion gesture recognition algorithms are shown in Table 2 and Figure 3.

From Figure 3, we can see that the improved motion posture recognition effect of the multiretention convolution algorithm is significantly higher than the other motion gesture recognition algorithms.

The video-based behavior monitoring method is to visually monitor the user's behavior with the help of a camera and use the video image recorded by the camera to identify the user's behavior. Images are divided into two-dimensional images and three-dimensional images. Generally, a single camera generates a two-dimensional image, and multiple cameras can synthesize a three-dimensional image. The different image features can divide the research direction into two-dimensional video image-based behavior recognition and three-dimensional video image-based behavior recognition. 
TABLE 1: Network fusion experiment results.

\begin{tabular}{lcccr}
\hline & Average & Model weights & Category weight & Model and category weights \\
\hline UCF101 & 94 & 95 & 95 & 96 \\
HMDB51 & 69 & 71 & 70 & 72 \\
\hline
\end{tabular}

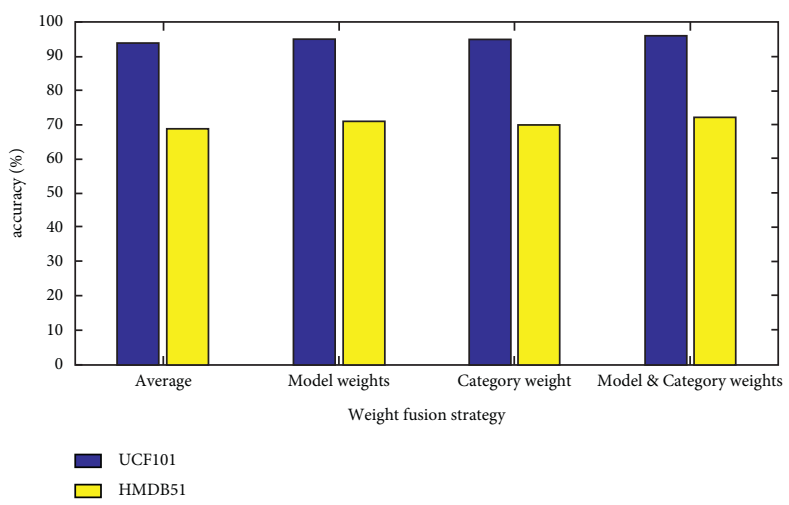

Figure 1: Network fusion experiment results.

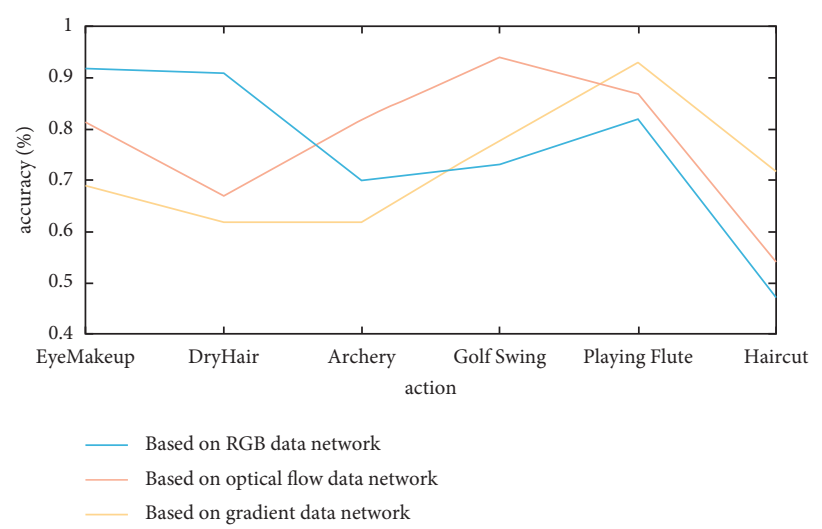

FIGURE 2: Classification results between different networks.

TABle 2: Comparison of experimental results of different motion gesture recognition algorithms.

\begin{tabular}{lcccccc}
\hline & IDT & Two-stream & TSN & C3D & LSTM & Multistream \\
\hline UCF101 & 87 & 89 & 95 & 86 & 94 & 96 \\
HMDB51 & 62 & 60 & 70 & 65 & 67 & 72 \\
\hline
\end{tabular}

4.2.2. Analysis of Experimental Results of Behavior Prediction Algorithm. Although the video-based behavior recognition method is the most direct monitoring method that everyone thinks, the video information collected by the camera has higher requirements for the environment. When the light is not strong or the coverage area of the camera is not large, the quality of the video image obtained by the device is relatively high. From the perspective of life, this method of real-time video monitoring of users is more difficult to achieve. Users who want to keep their own private space at home are generally reluctant to arrange multiple cameras at home.

In Figure 4, the accuracy of action classification using only the MLP method is very poor, and the use of ResNet deep to global characters of the image for action categorization can already achieve better accuracy, but ResNet can only extract the limited useful features; finally, the multifeature joint discriminant method in this paper can achieve higher accuracy. The test results of the behavior prediction methods are seen in Figure 4.

Since prediction algorithms combined with migration learning theory would necessarily perform longer algorithms based on a single dataset (CONVEX and SVM algorithms) and multisource-based algorithms (TrAdaBoost), the execution time test focused on the comparison between multiple source migration algorithms, namely, MS-TrAdaBoost, MO-TrAdaBoost, and IMO-TrAdaBoost algorithms. In 


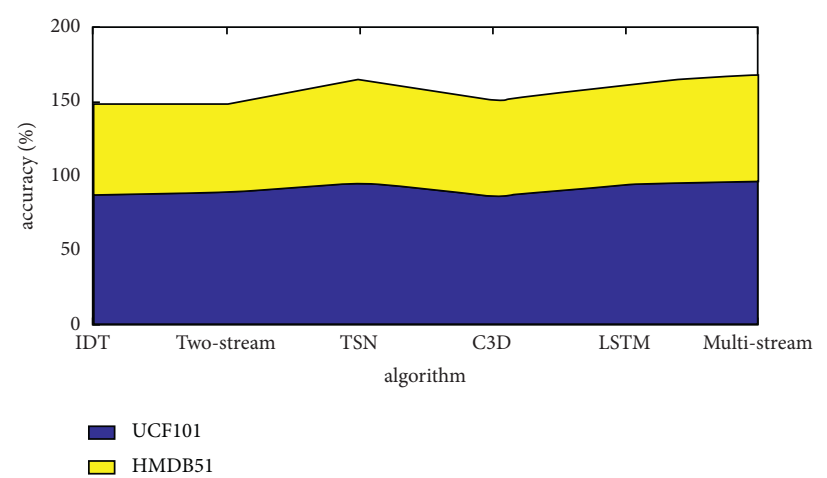

Figure 3: Comparison of experimental results of different motion gesture recognition algorithms.

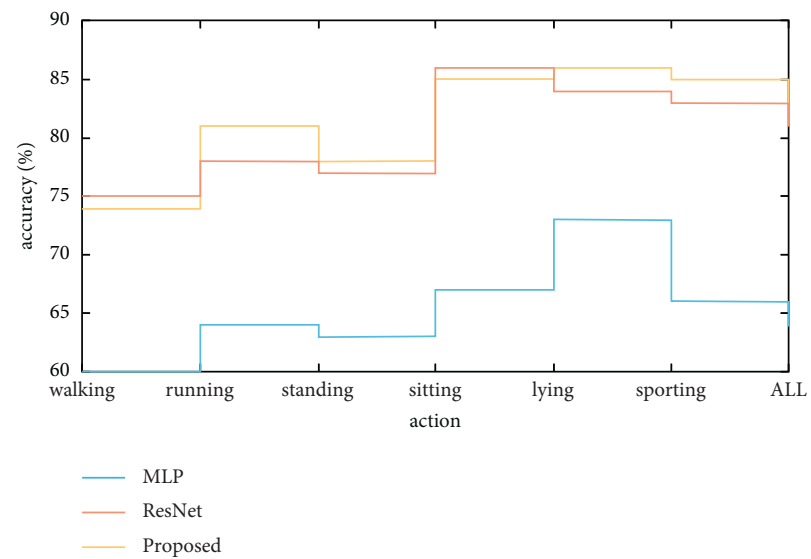

FIGURE 4: Experimental results of the behavior prediction algorithm.

addition, according to the characteristics and domain characteristics of the dataset in cultural modeling, the evaluation criteria used in the effective experiments have the accuracy, recall, recall, and $F$ values.

\section{Conclusions}

The low-cost characteristics of sensors and their specific advantages in wireless sensor networks have made them widely used in user behavior recognition. Many researchers use sensors to detect user behavior. These uses, according to their use and measurement methods, can be roughly divided into three types: portable devices, location-based, and nonintrusive devices. Based on the wireless sensor network, this paper focuses on solving the problems and difficulties in related key technologies as the main research line and discussions. The research content is mainly for indoor moving human targets and proposes a mixed Gaussian model based on the Ronski function. The moving target detection algorithm better realizes the complete detection of moving targets and the suppression of smear phenomenon; a human posture recognition algorithm based on multifeature fusion and image similarity is proposed for rapid human posture recognition, finally, to verify the effectiveness of the proposed algorithm, to achieve real-time detection and recognition of video. The results show that the improved algorithm in this paper can achieve the expected design goals, the detection effect of moving targets is better, the accuracy of human body gesture recognition is higher, and the experimental effect based on the standard video library is better. However, there are still certain limitations and shortcomings, and there is still room for improvement to solve the complex situation in the actually captured video. Nonintrusive behavior monitoring means that, in the process of collecting behavior data from users, the user's daily life is not disturbed, the user's privacy is not leaked, and the user's living habits are analyzed through the collected data. The nonintrusive way is generally to collect data by installing sensors on the indoor floor, roof, or object. This method is different from video equipment, portable equipment, and location-based methods. It is triggered by analyzing each sensor. Time and space information for user identification is as follows:

(1) Wireless sensor networks are very similar to selforganizing mobile networks. Both of these networks are wireless networks without infrastructure. But there is a clear difference between the two. This is mainly manifested in the following. The main purpose of mobile network design is to provide highquality and high-performance services and provide maximum bandwidth utilization in high-mobile environments. In most wireless sensor networks, 
sensor nodes usually do not move after deployment and can be deployed in environments inaccessible to humans.

(2) This paper is based on the situation that the current human pose recognition algorithm extracts single feature information and does not have good generalization ability. A human behavior acknowledgment method relying on a multistream job is proposed. This paper proposes two weighted fusion methods, which are model-based weight fusion and category-based weight fusion. Test results show that the algorithm results in a greater performance improvement. Exercise determines health, monitoring daily exercise rules helps to improve personal health index, and behavior recognition plays a vital role in it. Research on the field of behavior recognition has been going on for many years, and with the continuous innovation of science and technology, its research direction has also expanded in a wide range. The original behavior recognition research mainly focused on detecting and guiding personal health. Nowadays, behavior recognition is often used as a basic service. Through the identification of individual behavior status and the statistics of behavior status, it provides corresponding services for upperlevel applications.

(3) In this paper, through the improvement of the gesture recognition network, a new fully convolutional network branch is added for the segmentation of the character mask. By training such an end-toend network, it can be used for both gesture recognition and semantic segmentation. The advantage of such a network is that it does not require additional preprocessing like the background difference method. It has strong generalization applicable in any scene. It can classify pixels and segment the foreground of the character to avoid background interference. This article mainly establishes posture models and classifies common indoor postures. Postures mainly include walking, jumping, and running. A total of 3716 valid samples for each pose are selected from 12,890 sample images in the standard video library, and a multiclass SVM-based classifier is trained by extracting the feature vectors of the valid samples. In addition, in order to overcome the limitation and uncertainty of using the classification result of a single frame as the final recognition result, this paper proposes a secondary classification algorithm based on image similarity. The algorithm is based on the correlation between the video frames classified by the single-frame SVM for the first time, which improves the accuracy of gesture recognition while ensuring real-time performance.

(4) In this paper, we use MLP to extract local features from joint points and ResNet to extract global features from cut images. A multifeature joint learning behavior discrimination algorithm is proposed. This algorithm uses local features extracted by MLP which does not depend on the underlying body segmentation and tracking, is not very sensitive to noise and occlusion problems, and effectively improves the accuracy of behavior discrimination. The source organization screening algorithm based on attribute division and clustering is proposed to improve the effect of behavior prediction. We select the source organizations with a large correlation with the target task before knowledge migration, only making migration predictions on this part of the source organization, reducing the number of source organizations participating in iterative training, and thus reducing the time cost during training. Moreover, because the screening is based on the inherent nature of the organization, it overcomes the shortcomings of the traditional screening method based on data validation, solves the problem of source organization screening failure, and improves the prediction effect.

(5) The target monitored in this paper is a separate individual, the algorithm is easy, because of the lack of other target interference factors, but in real life, there are generally multiple monitoring targets, because of the increase in the monitoring target, and the algorithm complexity also needs to improve and to solve problems, including the mutual occlusion between targets and multitarget behavior correlation, which requires further study and improvement in the future.

\section{Data Availability}

This article does not cover data research. No data were used to support this study.

\section{Conflicts of Interest}

The authors declare that they have no conflicts of interest.

\section{Acknowledgments}

This work was supported by the Youth Fund Project of Wuhan Donghu University: Research on safety early warning system for the elderly based on data fusion (2021dhzk005).

\section{References}

[1] X. X. Zhu, D. Tuia, L. Mou et al., "Remote sensing: a comprehensive review and list of resources," IEEE Geoence and Remote Sensing Magazine, vol. 5, no. 4, pp. 8-36, 2017.

[2] B. Bansal, "Gesture recognition: a survey," International Journal of Computer Applications, vol. 139, no. 2, pp. 8-10, 2016.

[3] W. Shen, M. Zhou, F. Yang et al., "Multi-crop convolutional neural networks for lung nodule malignancy suspiciousness classification," Pattern Recognition, vol. 61, no. 61, pp. 663-673, 2017. 
[4] D. Holden, J. Saito, and T. Komura, "A deep learning framework for character motion synthesis and editing," ACM Transactions on Graphics, vol. 35, no. 4, pp. 1-11, 2016.

[5] A. Kamilaris and F. X. Prenafeta-Boldú, "Deep learning in agriculture: a survey," Computers and Electronics in Agriculture, vol. 147, no. 1, pp. 70-90, 2018.

[6] J. Han, D. Zhang, G. Cheng, N. Liu, and D. Xu, "Advanced deep-learning techniques for salient and category-specific object detection: a survey," IEEE Signal Processing Magazine, vol. 35, no. 1, pp. 84-100, 2018.

[7] W. Wang, M. Zhang, G. Chen, H. V. Jagadish, B. C. Ooi, and K.-L. Tan, "Database meets deep learning: challenges and opportunities," ACM SIGMOD Record, vol. 45, no. 2, pp. 17-22, 2016.

[8] H. J. Escalante, I. Guyon, V. Athitsos, P. Jangyodsuk, and J. Wan, "Principal motion components for one-shot gesture recognition," Pattern Analysis and Applications, vol. 20, no. 1, pp. 167-182, 2017.

[9] K. Martin Sagayam and D. Jude Hemanth, "Hand posture and gesture recognition techniques for virtual reality applications: a survey," Virtual Reality, vol. 21, no. 2, pp. 1-17, 2016.

[10] Y. Zou, J. Xiao, J. Han, K. Wu, Y. Li, and L. M. Ni, "GRfid: a device-free gesture recognition system using cots rfid device," IEEE Transactions on Mobile Computing, vol. 16, no. 2, p. 1, 2016.

[11] En-feng Hsu, M.-tsan Kao, and Yu-hao Huang, "Gesture recognition apparatus and complex optical apparatus," Perspectives in Education, vol. 22, no. 2, pp. 101-114, 2017.

[12] Y. Wu, S. Yu, and M. Yang, "High-performance gesture recognition system," Sensors and Materials, vol. 31, no. 3, p. 923, 2019.

[13] A. Ankith and E. Sasikala, "A survey on hand gesture recognition systems," International Journal of Computer Applications, vol. 180, no. 30, pp. 17-20, 2018.

[14] W. Pan and Q. Yang, "Transfer learning for behavior prediction," Intelligent Systems IEEE, vol. 31, no. 2, pp. 86-88, 2016.

[15] S. Qamar, H. Jin, R. Zheng, and P. Ahmad, "Multi stream 3D hyper-densely connected network for multi modality isointense infant brain MRI segmentation," Multimedia Tools and Applications, vol. 78, no. 18, pp. 25807-25828, 2019.

[16] W. Elsayed, M. Elhoseny, S. Sabbeh, and A. Riad, "Selfmaintenance model for wireless sensor networks," Computers \& Electrical Engineering, vol. 70, pp. 799-812, 2018.

[17] S. N. Mohanty, E. L. Lydia, M. Elhoseny, M. M. G. Al Otaibi, and K. Shankar, "Deep learning with LSTM based distributed data mining model for energy efficient wireless sensor networks," Physical Communication, vol. 40, Article ID 101097, 2020, In press.

[18] M. Thom and F. Gritschneder, "A theory for rapid exact signal scanning with deep multi-scale convolutional neural networks," Ieee Transactions on Signal Processing, vol. 65, no. 5, pp. 1235-1250, 2015.

[19] J. Fei, T. Rui, Y. Zhou, and F. Zhao, "Multi-input convolutional neural network based on gradient," Guangdian Gongcheng/opto Electronic Engineering, vol. 42, no. 3, pp. 33-38, 2015.

[20] M. Xin, Y. Zhang, S. Li, L. Zhou, and W. Li, "A locationcontext awareness mobile services collaborative recommendation algorithm based on user behavior prediction," International Journal of Web Services Research, vol. 14, no. 2, pp. 45-66, 2017.
[21] L. Bhansali and M. Narvekar, "Gesture recognition to make umpire decisions," International Journal of Computer Applications, vol. 148, no. 14, pp. 26-29, 2016.

[22] H. Song and M. Brandt-Pearce, "A 2-D discrete-time model of physical impairments in wavelength-division multiplexing systems," Journal of Lightwave Technology, vol. 30, no. 5, pp. 713-726, 2012.

[23] R. Craciunescu, A. D. Mihovska, and S. Kyriazakos, "Non audio-video gesture recognition system," Acta Veterinaria Scandinavica, vol. 8, no. 2, pp. 98-110, 2016. 